\title{
Hydatid cyst of the thyroid gland with tracheal fistula: A case report and review of the literature
}

\author{
TIEMIN JIANG, QIANG GUO, BO RAN, RUIQING ZHANG, TUERGANAILI AJI and YINGMEI SHAO
}

\begin{abstract}
Hepatobiliary and Hydatid Department of The Digestive and Vascular Surgery Centre, Xinjiang Key Laboratory of Echinococcosis, The First Affiliated Hospital, Xinjiang Medical University, Xinjiang, Urumqi 830054, P.R. China
\end{abstract}

Received July 20, 2018; Accepted January 24, 2019

DOI: $10.3892 /$ etm.2019.7620

\begin{abstract}
Hydatid disease, also known as echinococcal disease, is a zoonotic disease caused by Echinococcus infection. Hydatid cysts of the thyroid are rare. The present case study reports on a patient with hydatid cyst in the thyroid with tracheal fistula. A 54-year-old male patient without any history of exposure to farm animals was hospitalized due to fever and productive cough with occasional hydatid daughter cyst. The patient was diagnosed with a hydatid cyst of the thyroid gland with tracheal fistula by relevant examination. An operation was performed to remove the hydatid cyst and to repair the fistula. The patient took oral albendazole after surgery to prevent hydatid recurrence. Operative recovery was uneventful and the patient resumed his normal activities. Prompt diagnosis and an appropriate surgical treatment prevented a potentially fatal outcome. Furthermore, the characteristics of thyroid cystic echinococcosis, and its diagnosis and treatment in the present case and other cases reported in the literature were summarized and reviewed. Although the thyroid gland is rarely affected, hydatid cyst disease should be considered as a differential diagnosis of cystic lesions of the thyroid gland in patients living in regions where hydatid cyst disease is endemic.
\end{abstract}

\section{Introduction}

Echinococcosis caused by Echinococcus granulosus is a common type of zoonosis in farming and grazing areas and is distributed worldwide (1). The major organ affected is the liver, but extrahepatic disease is also possible. Only few cases of thyroid cystic hydatid disease have been reported worldwide.

Correspondence to: Dr Yingmei Shao or Dr Tuerganaili Aji, Hepatobiliary and Hydatid Department of The Digestive and Vascular Surgery Centre, Xinjiang Key Laboratory of Echinococcosis, The First Affiliated Hospital, Xinjiang Medical University, 393 Xinyi Road, Xinshi, Xinjiang, Urumqi 830054, P.R. China

E-mail: syingmei1@163.com

E-mail: tuergan78@sina.com

Key words: hydatid cysts, thyroid gland, tracheal fistula
However, the possibility of cystic hydatid disease cannot be ignored in those areas with a high incidence of hydatid disease.

Echinococcosis in rare sites, including the thyroid, is mostly misdiagnosed, predominantly due to its rarity. It is at times diagnosed as cystic echinococcosis during surgery and even treated on the basis of the incorrect diagnosis. For echinococcosis in rare locations, including thyroid cystic echinococcosis, various countries and regions have implemented different treatment modalities, such as total thyroidectomy, partial thyroidectomy and fine needle aspiration cytology (FNAC) $(2,3)$.

The present study presents a case of hydatid cyst-tracheal fistula in the thyroid. Furthermore, the literature on cystic echinococcosis of the thyroid gland was reviewed to summarize the characteristics of thyroid cystic echinococcosis, and compare its diagnosis and treatment among studies.

\section{Case report}

A 54-year-old male patient without any history of exposure to farm animals presenting with a gradual swelling on the left side of the lower part of the neck over a period of two years was admitted to the First Affiliated Hospital of Xinjiang Medical University in November 2013. The swelling was first noticed $\sim 20$ years previously. Furthermore, the patient presented with fever and productive cough with occasional hydatid daughter cyst for 2 months. Difficulty in breathing appeared twice and the symptoms were alleviated after oxygen therapy and intramuscular injection of dexamethasone. During physical examination, a non-tender, soft and fluctuant mass was palpable on the left lateral side of the neck. The size of the mass was measured as $7 \times 4 \mathrm{~cm}$. The computed tomography (CT) scan of the abdomen and pelvis indicated no evidence of hydatid cyst. Bronchoscopy examination revealed an ulcer just $2 \mathrm{~cm}$ below the glottis and this ulcer was $8 \mathrm{~mm}$ in diameter. The hydatid immunology test was positive. CT (Fig. 1A) and magnetic resonance imaging (MRI; Fig. 1B) revealed hydatid on the two sides of the thyroid.

The patient received surgery for excision of the internal capsule. During surgery, the hydatid cyst was removed, while the surrounding area was protected. A fistula of $1 \mathrm{~cm}$ in diameter was identified between the trachea and cyst (Fig. 2), and the fistula was repaired. Two drainage tubes were placed in the operation area. The post-operative pathological diagnosis was echinococcosis granulosa of the thyroid (Fig. 3). The fistula orifice between the hydatid residual cavity and the trachea 
A

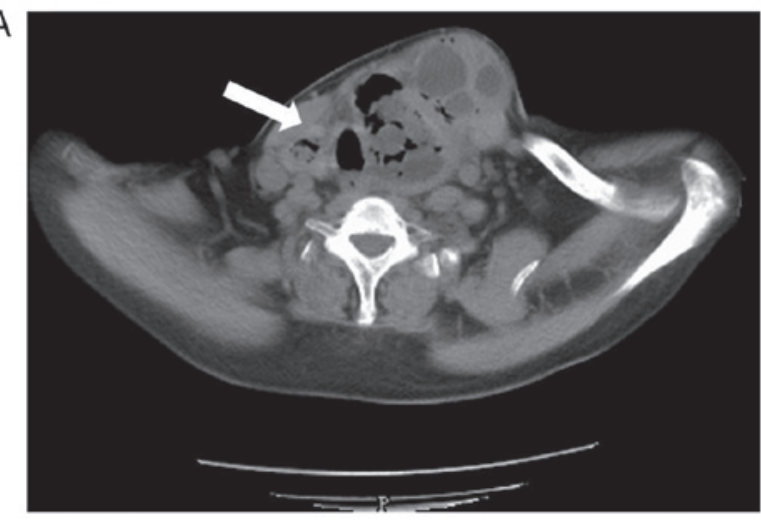

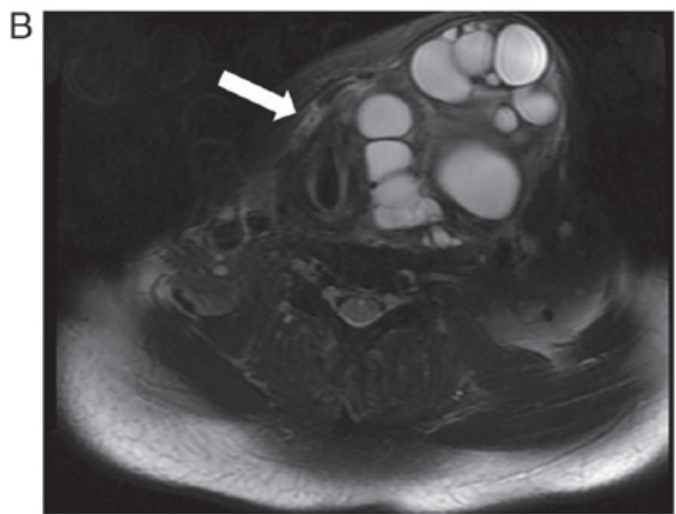

Figure 1. Pre-operative imaging analysis. (A) Pre-operative CT. (B) Pre-operative MRI. The CT and MRI revealed hydatid (white arrows) on the left and right sides of the thyroid. CT, computed tomography; MRI, magnetic resonance imaging.

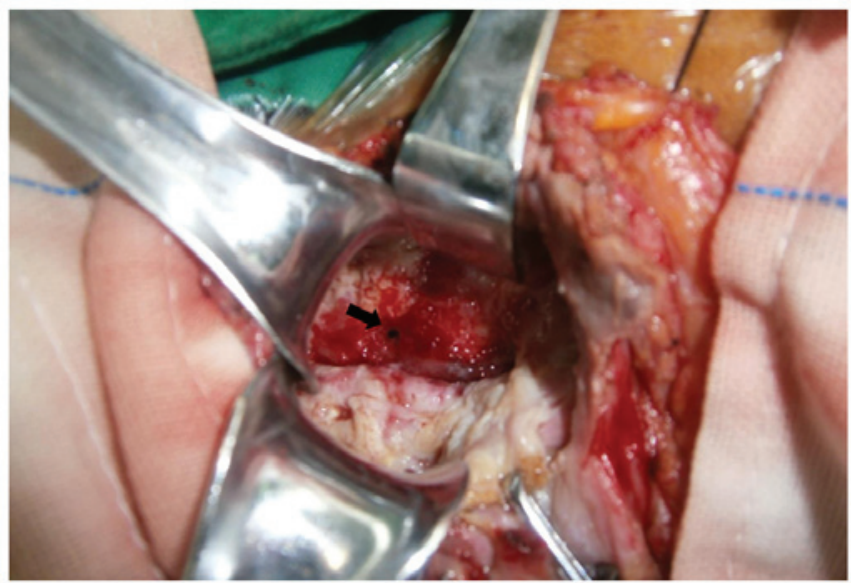

Figure 2. Intra-surgical observation after removal of the cyst. A fistula (diameter, $1 \mathrm{~cm}$; black arrow) was detected between the trachea and cyst.

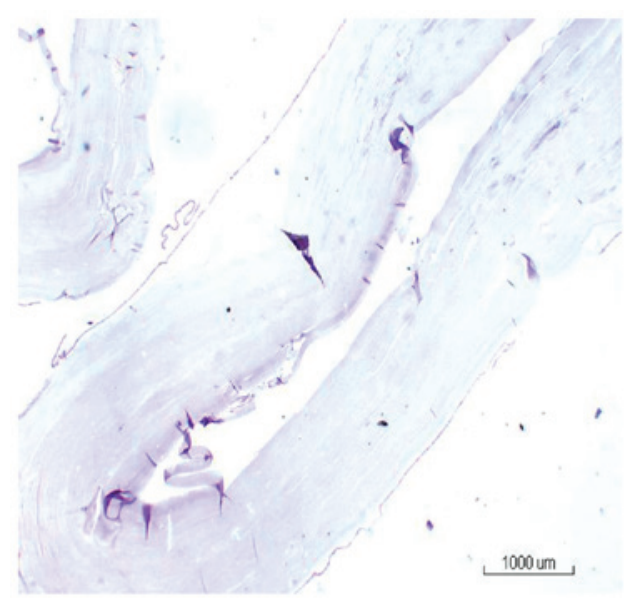

Figure 3. Post-operative pathological analysis (scale bar, 1,000 $\mu \mathrm{m}$ ). The fine-needle aspirate was stained with hematoxylin $\&$ eosin. The diagnosis of the echinococcosis granulosa of the thyroid was confirmed.

was still visible on $\mathrm{CT}$ at 5 days after the surgery (Fig. 4). Inflammation in the lung was also still visible. A small amount of gas $\left(\sim 50 \mathrm{~cm}^{3}\right)$ appeared in the drainage bag of the operated area when the patient coughed. The gas volume was generally

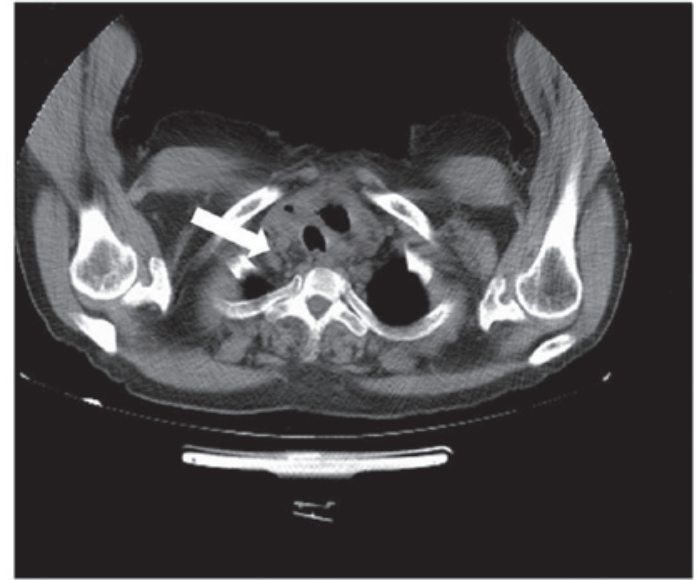

Figure 4. Post-operative computed tomogram at 5 days after the surgery. The fistula orifice (white arrow) was still visible.

low. Due to the low quantity of the gas, the tube was pulled out on the fifth day after the operation. The fistula had disappeared in the review CT scan at 5 days after the surgery. The patient continued to receive albendazole therapy $(15 \mathrm{mg} / \mathrm{kg} /$ day $)$ for 1 year post-operatively. No recurrence was observed in the thyroid during the 54-month post-operative follow-up period.

\section{Discussion}

Hydatid disease is a parasitic infection with worldwide prevalence (4). A hydatid cyst of Echinococcus granulosus may develop in any part of the body (5-8). Although it mostly tends to form cysts in the liver $(75 \%)$ or lung $(15 \%)$, other organs of the body, including the brain, heart, bones, muscle, kidney and pancreas, may also be affected (8-11). While thyroid involvement of echinococcosis is rare, thyroid hydatid with tracheal fistula is even rarer.

A high circulatory flow rate in the thyroid gland may have an important role in the development of the thyroid hydatid cyst $(6,8,9)$. It remains elusive how the relatively high thyroid blood flow is paralleled with a disproportionately low incidence of thyroid cyst disease, but one theory indicates that the relatively small caliber of the thyroid arteries together with their position at a right angle to the carotids is an anatomical arrangement that 
is thought to offer the thyroid gland a certain measure of protection against the invading parasite (4).

Patients with thyroid hydatid cyst are at times asymptomatic. A few hydatid patients present with symptoms including hoarseness and dyspnea, which are caused by the compression of surrounding tissues during the growth of the hydatid. When the patients present with symptoms of cough and expectoration, hydatid-bronchial fistula should be considered. If the patient has coughed-up discharge of cystic fluid and even daughter cyst of hydatid, hydatid-tracheal fistula may be diagnosed.

Ultrasound, CT and MRI are of great value in the diagnosis of thyroid primary disease. Thyroid hydatid should be distinguished from thyroid cyst. The imaging findings of thyroid hydatid and thyroid cyst are similar. Through analyzing the patient's epidemiological history (such as history of exposure to farm animals or history of living in an area with high prevalence) and history of hepatic hydatid disease, and considering the immunological results, thyroid hydatid may be diagnosed.

At present, the management of choice for hydatid disease is early detection. Surgical intervention includes the excision of the cyst and a part of the involved adjacent thyroid gland (4). It is preferred to not excise the thyroid or excise the thyroid as little as possible, as this avoids post-operative hypothyroidism. Surgery should be performed carefully to prevent extrusion of the hydatid fluid or sac into the trachea. At present, surgical treatment is the first choice for treating hydatid disease. However, drug treatment prior to and after surgery may improve the cure rate and reduce the recurrence rate of the disease (12).

A literature search was performed in the PubMed, Medline and Google Scholar databases by using key words, including 'Thyroid', 'Echinococcosis', 'Hydatid cyst' and 'Hydatid disease', in different combinations. Studies were limited to those in English and Chinese language. The abstracts were retrieved and reviewed for pre-selection, followed by retrieval of the full articles. A total of 18 articles $(4,7,13-28)$ with detailed descriptions were identified, and 21 cases were reported in them. Table I presents the detailed data of these studies. Based on the specific data in these studies, the diagnosis and treatment thyroid cystic echinococcosis was summarized and analyzed.

Of the 18 studies from 7 countries, 11 studies from Turkey reported on 14 patients, 2 studies from India reported on 2 cases and the remaining articles were from the USA, Morocco, Iran, Australia and China, with each study reporting on one case. The studies comprised a total of 21 patients (14 women and 7 men). The age at onset was 9-65 years with the median age of 30 years.

The major complaints of the patients with thyroid cystic echinococcosis were progressive enlarged neck mass, neck pain, hoarseness and difficulty in breathing. Most of the patients (14/21) presented with enlarged masses on the neck and had no specific symptoms. A total of 4 patients complained of hoarseness and 2 of neck pain, and 2 patients had dyspnea. Two patients presented with thyroid cystic lesions revealed through physical examination. The hydatid lesion itself appeared to cause no specific symptoms and the abovementioned complaints were mainly caused by compression of the increased hydatid lesions.

Pre-operative diagnosis is important for thyroid hydatid disease. The diagnostic methods include review of the patient history, physical examination, imaging examination, serological examination and FNAC diagnosis (29). The imaging diagnosis is considered to be the most important one $(24,30,31)$. The imaging diagnosis usually includes ultrasound, CT and MRI. Ultrasonography is effective in detecting the cystic lesions and is important for the pre-operative diagnosis (32). CT scan and MRI are usually complementary diagnostic tools. For multiple hydatid cysts, particularly in patients with a history of hydatid disease, the diagnosis is straightforward; however, for a less experienced clinician, it is difficult to identify a primary thyroid monocystic echinococcosis from a single cyst. The most common misdiagnoses are simple thyroid cysts. By ultrasound, the round and non-echo lesions, visible floating small light spots in the dark area and the 'sand bag syndrome' may be observed (33). The cystic wall is smooth and complete and exhibits a double-layer sign (34). Regarding the differential diagnosis, ultrasound is superior to CT and MRI (35). In addition, the patient's epidemiological history and information on whether hydatid disease is present in the liver are helpful for making a definite diagnosis. CT examination is able to accurately display the narrowing and displacement when the hydatid lesions involve the blood vessels and trachea (36), and may clearly display the association of the hydatid lesions with surrounding tissues (37), which is of significance in guiding pre-operative preparation and surgical planning. However, bronchial fistula is characterized by the presence of a shadow of gas density in the hydatid cavity. For larger fistula, CT and MRI may indicate the size and location of the fistula. Bronchoscopy may directly display the fistula and the location of the fistula in lesions generally presents as the most prominent site of the trachea.

FNAC is generally accepted in the clinical treatment of the single thyroid nodules (38). Although no study has reported any allergic reactions after FNAC, this technique is not recommended, as it may cause the diffusion of the cystic fluids or allergic reactions. Therefore, FNAC should be avoided when differential diagnosis of thyroid hydatid cysts is needed.

The immunological method is also an important modality for the diagnosis of echinococcosis, particularly as a differential diagnosis (39). The traditional Casoni intradermal test has a high false-positive rate and is overly sensitive. Immune disturbances seriously affect the follow-up after treatment and have been removed from the World Health Organization (WHO)'s Hydatid Disease Diagnosis and Treatment Guidelines (2001 edition) (3). In addition, the test strip and ELISA methods also have important roles in the screening for echinococcosis.

Among all cases analyzed, thyroid hydatid was a condition primary to the thyroid. Examination of patients (15/21) revealed that only the thyroid gland contained cystic hydatid and that no organs had any hydatid lesions. In general, the liver is the most susceptible and the first site of infection for echinococcosis (40). Among the 21 cases, only two were combined with hepatic cystic echinococcosis. One case was combined with hydatid in multiple sites, including the liver, lung and abdomen. One patient had lung hydatid 10 years previously, which was removed by surgery. One patient had hepatic hydatid 2 years previously, which was treated by surgery. In another case, there was no mention of whether any other organs exhibited any hydatid cysts.

Of the 21 cases, one patient received puncture treatment twice prior to surgery. Three patients underwent FNAC pathological examination in order to clarify the pre-operative diagnosis. One case from Turkey reported in 2010 (24) received 


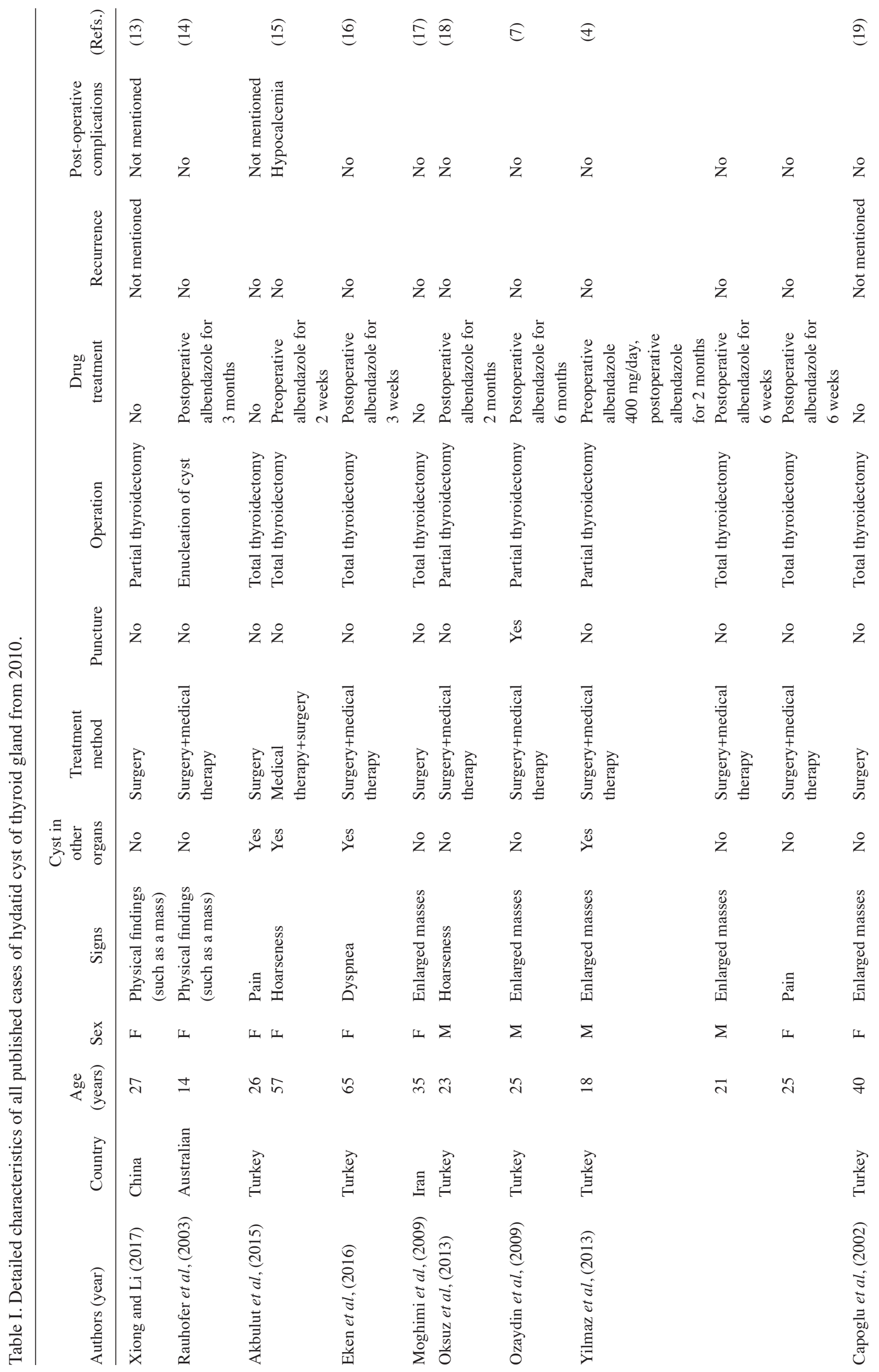




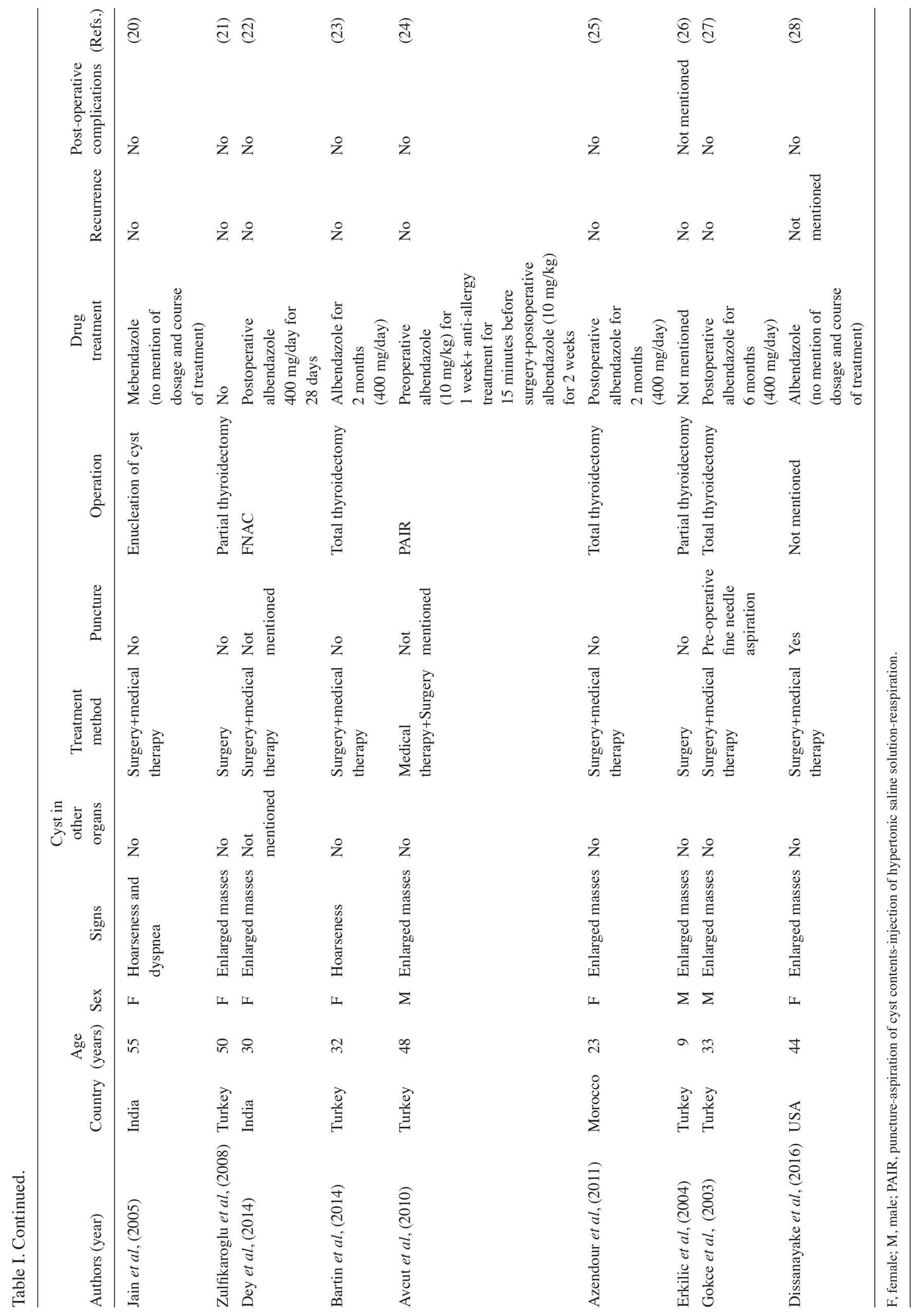


puncture-aspiration of cyst contents-injection of hypertonic saline solution-reaspiration (PAIR) treatment. In this case, two daughter cysts of the hydatid lesions were punctured, $35 \%$ of cyst fluid was aspirated and $20 \%$ hypertonic saline was injected into the cavity for $10 \mathrm{~min}$. The patient was subjected to post-operative albendazole treatment for 2 weeks and reported no complications during the treatment. During the 4-month follow-up, a 'pseudotumor appearance' without any fluid component or daughter vesicles was noted. In the case of the present study, surgical treatment was preferred, as PAIR technology may rather be suitable for monocystic hydatid cyst. For multiple hydatid cysts, hypertonic saline cannot completely destroy the capsule wall of the daughter cyst as well as inactivate the hydatid sac. However, spillage of the cyst fluids may also occur, which may even increase the risk of allergic reactions. Therefore, puncture treatment of the hydatid cysts is not recommended at our medical center. Among the reviewed literature, three studies $(19,20,22)$ also indicated that the hydatid puncture is considered to be associated with a high risk and is not recommended. Therefore, the safety and efficacy of PAIR remains controversial and requires further investigation.

Surgical treatment remains the preferred method for the treatment of thyroid hydatid cysts (41). Most of the patients underwent partial or complete thyroidectomy. The cases reviewed comprised 17 cases of thyroidectomy, including 9 cases of total thyroidectomy, 6 cases of ipsilateral partial thyroidectomy and 2 cases of hydatid cyst removal. One of the patients presented with hypocalcemia directly after total thyroidectomy and during the post-operative 4-year follow-up (42). The case reported in the present study only received excision of the hydatid and repair of the fistula orifice connected with the bronchus. Based on previous experience, for the excision of the thyroid gland, regardless of whether it is a total or an ipsilateral excision, the removal of the normal thyroid tissue may be unnecessary and easily causes other associated complications, including peripheral nerve or parathyroid damage. Even if no other complications occur, total resection of the thyroid may require lifelong treatment with thyroid hormones. In summary, the surgical removal of hydatid cysts followed by post-operative albendazole treatment is a safe and effective method.

A total of 5 patients with total thyroidectomy and 2 with ipsilateral thyroidectomy did not take any anti-hydatid drugs after the surgery, whereas the remaining 13 patients took anti-hydatid drugs after the surgery. In the reviewed studies, most patients were advised to take oral albendazole. Only one case from India reported in 2005 (20) was treated with oral mebendazole. Among the studies, a large difference in the duration of drug intake was noted; for instance, the duration of albendazole treatment was as short as 2 weeks in one case (15) and the maximum time was 6 months (27). The specific duration of taking the drug was not specified in two of the studies. The dose of albendazole was $400 \mathrm{mg} /$ day in 4 of the studies $(22,23,25,27)$ and $10 \mathrm{mg} / \mathrm{kg} /$ day based on the weight of the patient in one study (24).

At present, the preferred drugs for the treatment of echinococcosis are benzimidazole drugs, of which the representative drugs are mebendazole and albendazole $(43,44)$. The treatment effect of albendazole has been demonstrated to be significantly better than that of mebendazole $(3,12)$, and it has now replaced mebendazole and become one of the drugs of choice recommended by the
WHO (2,3). Albendazole is metabolized into albendazole and sulfoxide in the liver and exerts an anti-helminthic effect; it significantly inhibits the parasite's absorption of glucose, resulting in the exhaustion of glucose or inhibition of the fumarate reductase system. This mechanism impedes the formation of ATP, making parasites unable to survive and reproduce. As echinococcosis has a high recurrence rate (45) and each patient has a different extent of disease, the post-operative drug therapy for hydatidosis patients should be individualized based on the activity of the hydatid lesions and the specific circumstances of the patients. Even if a non-curative surgery is performed and the activity of the hydatid lesions is not high or even in a state of solid necrosis, the time for taking the drug may be reduced. For single-capsule, multi-ascendant and internal capsule collapse types, 3-12 months of post-operative drug therapy is recommended (45). It is more clinically significant to determine the dose according to the weight of the patient. Based on our experience, the total daily dose is $10-15 \mathrm{mg} / \mathrm{kg}$, half of which should be administered each morning and evening. An individual study also reported the administration of pre-operative prophylactic oral albendazole (24), and according to expert consensus, pre-operative treatment for 3-7 days is also recommended.

Despite significant advances in diagnostic research, as well as medical and surgical treatment, echinococcosis remains a challenging disease with high morbidity. Hydatid cyst in the thyroid is rare. The diagnosis of hydatid cyst in the thyroid should be considered in epidemic regions of hydatid disease. The diagnosis and treatment of thyroid cystic hydatid vary considerably among different regions, particularly the choice of surgical procedure and the duration and dosage of therapeutic drugs. At present, there is no uniform standard of treatment. In conclusion, the diagnosis and treatment of hydatid cyst in the thyroid reported in recent years were reviewed. The present study may enhance the understanding and awareness of hydatid cyst in the thyroid and provide a reference for clinicians.

\section{Acknowledgements}

Not applicable.

\section{Funding}

The present study was supported by the National Science Foundation of China (grant nos. 81560098 and 81660108) and Natural Science Foundation of Xinjiang Province (grant no. 2016D01C274).

\section{Availability of data and materials}

The datasets used and/or analyzed during the present study are available from the corresponding author on reasonable request.

\section{Authors' contributions}

TJ, BR, RZ and QG collected the data. TJ wrote the manuscript. YS and TA conceived and designed the present study, and revised the manuscript. All authors read and approved the final version of the manuscript. 


\section{Ethics approval and consent to participate}

The present study was approved by the ethics review board of the First Affiliated Hospital of Xinjiang Medical University (Xinjiang, China).

\section{Patient consent for publication}

Informed consent was obtained from the patient.

\section{Competing interests}

The authors declare that they have no competing interests.

\section{References}

1. McManus DP, Zhang W, Li J and Bartley PB: Echinococcosis. Lancet 362: 1295-1304, 2003.

2. Brunetti E, Kern P and Vuitton DA; Writing Panel for the WHO-IWGE: Expert consensus for the diagnosis and treatment of cystic and alveolar echinococcosis in humans. Acta Trop 114: $1-16,2010$

3. Guidelines for treatment of cystic and alveolar echinococcosis in humans. WHO Informal Working Group on Echinococcosis. Bull World Health Organ 74: 231-242, 1996 (In English, French).

4. Yilmaz M, Akbulut S, Sogutlu G, Arabaci E and Kayaalp C: Hydatid cyst of the thyroid gland: Report of three cases. Surg Today 43: 937-941, 2013.

5. Akbulut S, Senol A, Ekin A, Bakir S, Bayan K and Dursun M: Primary retroperitoneal hydatid cyst: Report of 2 cases and review of 41 published cases. Int Surg 95: 189-196, 2010.

6. Erbil Y, Barbaros U, Baspinar I, Deveci U, Deveci U, Kapran Y, Bozbora A, Aral F and Özarmağan S: Hydatid cyst of the thyroid gland: Two case reports. Infect Dis Clin Pract 13: 318-320, 2005.

7. Ozaydin I, Ozaydin C, Oksuz S and Yildirim M: Primary Echinococcus cyst of the thyroid: A case report. Acta Med Iran 49: 262-264, 2011.

8. Azendour I, Boulaich M, Ayoubi A, Oujilal A, Essakalli L and Kzadri M: Primary hydatid cyst of the thyroid gland. Int J Otolaryngol 2011: 713089, 2011.

9. Gökçe C, Patiroğlu T, Akşehirli S, Durak AC and Kelestimur F: Hydatic cyst in the thyroid gland diagnosed by fine-needle aspiration biopsy. Thyroid 13: 987-989, 2003.

10. Özerkan E, Gürçınar M, Sarıoğlu B and Aydınlıŏlu H: A case of cystic echinoccosis in thyroid gland: A very rare localisation of echinoccosis infection. Turk J Endocrinol Metabol 4: 181-183, 1999

11. Pérez PJA, Felmer EO, Carrasco EC, Gabrielli NM, Torrijos CC and Bastías NJA: Thyroid hydatid cyst: Report of one case. Rev Med Chil 136: 896-899, 2008 (In Spanish).

12. Senyüz OF, Yeşildag E and Celayir S: Albendazole therapy in the treatment of hydatid liver disease. Surg Today 31: 487-491, 2001.

13. Xiong W and Li L: A case of thyroid hydatid in Tibet, China. Contemp Med 28: 126, 2017 (In Chinese).

14. Rauhofer U, Prager G, Hörmann M, Auer H, Kaserer K and Niederle B: Cystic echinococcosis of the thyroid gland in children and adults. Thyroid 13: 497-502, 2003.

15. Akbulut S, Demircan F and Sogutcu N: Hydatid cyst disease of the thyroid gland: Report of two cases. Int Surg 100: 643-647, 2015

16. Eken H, Isik A, Balci G, Firat D, Cimen O and Soyturk M: A rare case of isolated cystic hydatid of thyroid gland. Medicine (Baltimore) 95: e2929, 2016.

17. Moghimi M, Kamrava SK, Asghari AM, Heshmatzade Behzadi A, Jalessi M, Naraghi MM and Ehteshamia Afshar E: Primary echisnococcal cyst in the thyroid gland: A case report from Iran. J Infect Dev Ctries 3: 732-734, 2009.

18. Oksuz S, Pektas E, Yavuz M, Aksungur N, Cayir Y and Akcay MN: An unusual cause of hoarseness: Hydatid cyst of the thyroid. Trop Biomed 30: 642-644, 2013

19. Capoğlu I, Unüvar N, Erdogan F, Yilmaz O and Caydere M: A hydatid cyst of the thyroid gland. J Int Med Res 30: 206-209, 2002.

20. Jain SK, Jamdade PT, Muneshwar SS, Ambulgekar VK and Panzade SM: Hydatid cyst of thyroid: An unusual cause of stridor. Indian J Otolaryngol Head Neck Surg 57: 80-81, 2005.
21. Zulfikaroglu B, Ozalp N, Keskek M and Koc M: Primary echinococcal cyst of the thyroid: Report of a case. Surg Today 38: 833-835, 2008

22. Dey AK, Mandal PK, Dutta A, Pramanik S, Maji S, Chakraborti and Dasgupta S: A case of hydatid cyst of thyroid gland. Asian J Med Sci 5: 143-145, 2014

23. Bartın MK, Yılmaz EM, Arslan H, Tekeli AE and Karataş S: A case of primary hydatid cyst in the thyroid gland. Ulus Cerrahi Derg 31: 94-95, 2014.

24. Avcu S, Unal O, Kotan C, Oztürk M and Ozen O: Submandibular and thyroid gland involvement of hydatid cysts: A very rare association with percutaneous treatment. Diagn Interv Radiol 16: 251-254, 2010.

25. Azendour I, Boulaich M, Ayoubi A, Oujilal A, Essakalli L and Kzadri M: Primary hydatid cyst of the thyroid gland. Int J Otolaryngol 2011: 713089, 2011.

26. Erkiliç S, Ozsaraç C, Koçer NE and Bayazit YA: Hydaitd cyst of the thyroid gland in a child. Int J Pediatr Otorhinolaryngol 68: 369-371, 2004.

27. Gökçe C, Patiroğlu T, Akşehirli S, Durak AC and Keleştimur F: Hydatic cyst in the thyroid gland diagnosed by fine-needle aspiration biopsy. Thyroid 13: 987-989, 2003.

28. Dissanayake PI, Chennuri R and Tarjan G: Fine-needle aspiration diagnosis of primary hydatid disease of the thyroid; first reported case in the USA. Diagn Cytopathol 4: 334-337, 2016.

29. Kammerer WS and Schantz PM: Echinococcal disease. Infect Dis Clin North Am 7: 605-618, 1993.

30. Gharbi HA, Hassine W, Brauner MW and Dupuch K: Ultrasound examination of the hydatic liver. Radiology 139: 459-463, 1981.

31. Yaliniz H, Tokcan A, Salih OK and Ulus T: Surgical treatment of cardiac hydatid disease: A report of 7 cases. Tex Heart Inst J 33: 333-339, 2006.

32. Versaci A, Macri A, Terranova M, Galipò S, Barbera A, Speciale G and Famulari C: Primary echinococcosis of the thyroid gland: Apropos of a case. G Chir 18: 427-431, 1997 (In Italian).

33. Song FL, Han SH, Fang J and Zhang L: Diagnostic value of hepatic hydatid cyst with CT and MRI. J Pract Radiol, 2006.

34. Zhang HX and H.N. F: Clinical diagnosis and treatment of liver cystic echinococcosis. Chin J Endemiol 34: 309-312, 2015.

35. WHO Informal Working Group: International classification of ultrasound images in cystic echinococcosis for application in clinical and field epidemiological settings. Acta Trop 85: 253-261, 2003.

36. Engin G, Acunas B, Rozanes I and Acunas G: Hydatid disease with unusual localization. Eur Radiol 10: 1904-1912, 2000.

37. Zhao YP, Liu WY, Wang J, Liu XL and Dang J: The value of CT score in predicting surgical methods and difficulty of hepatic cystic echinococcosis. Chin J Radiol 45: 55-59, 2011 (In Chinese).

38. Yoo WS and Chung HK: Recent advances in autoimmune thyroid diseases. Endocrinol Metab (Seoul) 31: 379-385, 2016.

39. Kern P, Menezes da Silva A, Akhan O, Mullhaupt B Vizcaychipi KA, Budke C and Vuitton DA: The echinococcoses: Diagnosis, clinical management and burden of disease. Adv Parasitol 96: 259-369, 2017

40. Grozavu C, Ilias M and Pantile D: Multivisceral echinococcosis: Concept, diagnosis, management. Chirurgia (Bucur) 109: 758-768, 2014.

41. Menezes da Silva A: Hydatid cyst of the liver-criteria for the selection of appropriate treatment. Acta Trop 85: 237-242, 2003.

42. Moghimi M, Kamrava SK, Asghari AM, Heshmatzade Behzadi A, Jalessi M, Naraghi MM and Ehteshamia Afshar E: Primary echinococcal cyst in the thyroid gland: A case report from Iran. J Infect Dev Ctries 3: 732-734, 2009.

43. Davis A, Dixon H and Pawlowski ZS: Multicentre clinical trials of benzimidazole-carbamates in human cystic echinococcosis (phase 2). Bull World Health Organ 67: 503-508, 1989.

44. Vutova K, Mechkov G, Vachkov P, Petkov R, Georgiev P, Handjiev S, Ivanov A and Todorov T: Effect of mebendazole on human cystic echinococcosis: The role of dosage and treatment duration. Ann Trop Med Parasitol 93: 357-365, 1999.

45. Wen H: Advancement of diagnosis and surgical treatment for hepatic echinococcosis. Zhong Hua Xiao Hua Wai Ke Za Zhi 10: 290-292, 2011 (In chinese).

This work is licensed under a Creative Commons Attribution-NonCommercial-NoDerivatives 4.0 International (CC BY-NC-ND 4.0) License. 